\section{Brazilian Journal \\ of Chemical \\ Engineering}

ISSN 0104-6632

Printed in Brazil www.scielo.br/bjce

(G)

\title{
ACETIC ACID PRODUCTION BY THE NEWLY ISOLATED PSEUDOMONAS SP. CSJ-3
}

\author{
Juan Wang ${ }^{1,2}$, Chenliang Hao ${ }^{3}$, Huijuan Huang ${ }^{1}$, \\ Wenjing Tang ${ }^{1}$, Jianbin Zhang $^{1 *}$ and Cuiyan Wang ${ }^{*}$ \\ ${ }^{1}$ College of Chemical Engineering, Inner Mongolia University of Technology, Huhhot 010051, China \\ ${ }^{2}$ Jinxiang Management Committee of Ecological Wetland Scenic Area, Jining 272200, China \\ ${ }^{3}$ School of Chemical Engineering, Northwest University, Xi'an 710069, China
}

(Submitted: March 26, 2016; Revised: August 19, 2016; Accepted: November 21, 2016)

\begin{abstract}
A fermentative acetic acid producing strain, named CSJ-3, was isolated from sludge and identified as Pseudomonas sp. on the basis of $16 \mathrm{~S}$ rRNA gene analysis. The effects of cultivation conditions on the production of acetic acid by Pseudomonas sp. CSJ-3 were investigated in batch fermentation, and the maximum yield of acetic acid reached up to $0.49 \%$ during $30 \mathrm{~h}$ cultivation under the optimum growth condition, including fermentation temperature of $37.0^{\circ} \mathrm{C}$, fermentation $\mathrm{pH}$ of 4.50 , ethanol concentration of $8 \%$, carbon source (glucose) amount of $10 \mathrm{~g} / \mathrm{L}$, and rotation speed of $120 \mathrm{r} / \mathrm{min}$. When ultraviolet (UV) irradiation was used for the mutation in culture medium to improve the yield of acetic acid, the results showed that the yield of acetic acid reached up to $0.59 \%$. UV and FTIR confirmed that acetic acid was the major fermented product.
\end{abstract}

Keywords: Acetic acid; Fermentation; Bioconversion; Optimization

\section{INTRODUCTION}

Acetic acid that can be produced by geological and biological processes in natural environments is an important raw material in the organic chemical industry and is also the suitable carbon source for many microorganisms (Sievers et al., 1992; Trcek et al., 1997; Sokollek et al., 1998; Schüller et al., 2000; Trcek et al., 2000, 2015). Some microorganisms have the capability to degrade organic compounds to produce acetic acid so that the application of microorganisms for the production of acetic acid has gained tremendous attention. The production of acetic acid using microorganisms is a promising method using low value waste as raw materials (organic waste and biomass) (Jin et al., 2000; Jin et al., 2002) to produce important chemicals due to the lower requirements of energy supply. In anaerobic digestion from various floras, acetic acid bacteria play an important role in the functional niche
(Wang et al., 2013). Fermentative acidogenic microflora can produce propionic acid, butyric acid, and ethanol; however, acetic acid bacteria can lead them to further degrade into acetic acid and carbon dioxide or hydrogen through direct utilization of methanogenic microflora (Lange et al., 2001; Pratap et al., 2001; McHugh et al., 2003; Ge et al., 2006). Anaerobic fermentation with low cost substrates rich in carbohydrates, such as organic wastes or agricultural residues, is one promising method to produce acetic acid. However, the application of Pseudomonas sp. to produce acetic acid has never been reported so far.

Currently, it is difficult to decide the culture conditions for supporting the maximum activity of acetic acidproducing strain. Therefore, UV-induced mutagenesis is a frequently-used and effective method for strain breeding, because the pyrimidine bases have strong UV absorption (Xu et al., 2010). When UV light is absorbed, the neighboring double thymines in the DNA chain will 
form thymine dimer resulting in the mutation. When the reproduction of DNA begins, the site of the thymine dimer will possibly run over and the gap will be left if it is a single thymine dimer, which can make the false bases inserted to cause the mutation of AT-GC (Kulpa et al., 1986). So, UV-induced mutagenesis can be used in this work for an increase in the yield of acetic acid.

Previous studies have shown that cultivation conditions or operational parameters can significantly affect the growth of cells and the yield of acetic acid (Jin et al., 2007). The aim of this study was to isolate, characterize and identify an acetic acid-producing bacterium and evaluate its acetic acid-producing capacity. In order to determine the optimal fermentation conditions for maximum yield of acetic acid, the effects of culture temperature, fermentation $\mathrm{pH}$, substrate concentration, carbon source type and amount, and inoculum amount on the yield of acetic acid were investigated. In the present, a breeding method was developed for improving the acetic acid-producing activity of Pseudomonas sp. CSJ-3 by applying UV irradiation in combination with cultivation. Taking advantage of mutation induced by UV irradiation accelerates improvement of strains that are hard to cultivate in pure cultures. UV and FTIR spectra confirmed that the major fermented product was acetic acid.

\section{EXPERIMENTAL}

\section{Sample source}

The isolated sample of acid-forming strain was extracted form sludge, which comes from Ordos' Chuan Xiang garbage disposal Co. Ltd., China.

\section{Culture medium}

\section{Basic culture medium}

The basic culture medium was prepared as follows: glucose $10 \mathrm{~g} / \mathrm{L}$, yeast extract $10 \mathrm{~g} / \mathrm{L}, \mathrm{pH}=4.5$, ethanol $3 \%$ (v/v), and sterilized $\left(121^{\circ} \mathrm{C}, 20 \mathrm{~min}\right)$.

\section{Separation culture medium}

The separation culture medium was prepared as follows: glucose $10 \mathrm{~g} / \mathrm{L}$, yeast extract $10 \mathrm{~g} / \mathrm{L}, \mathrm{CaCO}_{3} 20$ $\mathrm{g} / \mathrm{L}$, agar $20 \mathrm{~g} / \mathrm{L}, \mathrm{pH}=6.0$, ethanol $3 \%(\mathrm{v} / \mathrm{v})$, and sterilized $\left(121^{\circ} \mathrm{C}, 20 \mathrm{~min}\right)$.

\section{Activating and fermentation culture medium}

The activating culture medium was prepared as follows: glucose $10 \mathrm{~g} / \mathrm{L}$, yeast extract $10 \mathrm{~g} / \mathrm{L}, \mathrm{pH}=4.5$, ethanol $7 \%$ (v/v), and sterilized $\left(121^{\circ} \mathrm{C}, 20 \mathrm{~min}\right)$.

\section{Slant preservation culture medium}

The slant preservation culture medium was prepared as follows: glucose $10 \mathrm{~g} / \mathrm{L}$, yeast extract $10 \mathrm{~g} / \mathrm{L}, \mathrm{CaCO}_{3} 10 \mathrm{~g} / \mathrm{L}$, agar $20 \mathrm{~g} / \mathrm{L}, \mathrm{pH}=6.0$, ethanol $3 \%(\mathrm{v} / \mathrm{v})$, and sterilized $\left(121^{\circ} \mathrm{C}, 20 \mathrm{~min}\right)$.

\section{Pretreatment of the sample}

$10 \mathrm{~g}$ sample was put into a one-neck flask containing aseptic water, was broken by glass balls, with controlled $\mathrm{pH}$ in the range of (4.0 to 6.0), and kept for $48 \mathrm{~h}$.

\section{Multiplication culture}

$15 \mathrm{~mL}$ clear liquid extracted from the pretreated sample was put into a flask containing $150 \mathrm{~mL}$ culture medium to produce the original bacterial sample. The enriched cell solution was obtained by adding the original bacteria into the culture medium for $48 \mathrm{~h}$ cultivation at $32.0^{\circ} \mathrm{C}$ and $120 \mathrm{r} / \mathrm{min}$.

\section{Isolation and purification}

The strain was inoculated on the separation culture medium and cultivated for $24 \mathrm{~h}$ at $32.0{ }^{\circ} \mathrm{C}$, and the strain's growth processes were observed and recorded. When a single colony was developed on the separation culture medium, the single colony was isolated from the separation culture medium and transferred to activating culture medium for cultivation, where the characteristics of colony, figures, sizes, and Gram's characteristics appeared. Then the strain was conserved as the pure strain and named as CSJ-3, which was cultivated on the slant preservation culture medium, maintained in the icebox at $4{ }^{\circ} \mathrm{C}$, and inoculated after 1-2 months.

\section{Identification of the strain}

The physiological \& biochemical characteristics of the strain were determined according to basic methods (Dong, 2001). The $16 \mathrm{~S}$ rRNA gene was amplified as previously described (Xing et al., 2006). The 16S rRNA gene sequence was aligned and identified against existing sequences in the GenBank database using the BLAST program.

\section{Experiments of preferred cultivation condition}

The separated strain was accessed by using the activating culture medium for $24 \mathrm{~h}$ at $32.0{ }^{\circ} \mathrm{C}$ and $120 \mathrm{r} /$ $\mathrm{min}$, and the cell concentration was controlled at $2.8 \times 10^{6} /$ $\mathrm{mL}$. Fermentation culture medium containing activated cell solution was used to perform the experiments of preferred cultivation conditions by changing fermentation conditions, such as the temperature, $\mathrm{pH}$, ethanol concentration, carbon source, inoculum volume, and rotation speeds of the sorting table. Meanwhile, an acid-base titration method was used to measure data and confirm the culture conditions as follows:

Acetic acid yield $(\%)=\left(\mathrm{V}_{\mathrm{t}}-\mathrm{V}_{0}\right) \times \mathrm{C}_{\mathrm{NaOH}} \times 60 /(5 \times 10.3 \mathrm{~V} /$ $[\mathrm{mL}]) \times 100 \% \quad$ (1) where $\mathrm{V}_{t}$ is volume of $\mathrm{NaOH}$ solution at $\mathrm{t} \mathrm{h}(\mathrm{mL}), \mathrm{V}_{0}$ is volume of $\mathrm{NaOH}$ solution at $0 \mathrm{~h}(\mathrm{~mL})$, $\mathrm{C}_{\mathrm{NaOH}}$ is the molar concentration of $\mathrm{NaOH}$ solution $(\mathrm{mol} / \mathrm{L})$, 60 is the molar mass of acetic acid $(\mathrm{g} / \mathrm{mol}), 5$ is the volume of fermentation culture medium $(\mathrm{mL}), 10.3$ denotes the constant of $1 \%$ ethanol in the media to acetic acid, and $\mathrm{V}$ denotes the percent of ethanol in the media. The uncertainty of acetic acid concentration was estimated to be $\pm 0.60 \%$. 


\section{Determination of growth curve}

The cell concentration of the strain was counted by a hemacytometer under the amplification of 640 times. The strain cells were dissociated into suspension in culture flasks, and the number of cells was counted by hemacytometry. The uncertainty of cell concentration was estimated to be $\pm 3 \%$. Then the growth curve was plotted on the counted data of cell concentration.

\section{Mutation breeding}

The experiment was carried out under the following conditions. The UV power was $15 \mathrm{~W}$, the distance was controlled at $45 \mathrm{~cm}$, and the radiation time was set at $(0,5$, $10,15,20,25,30,35$, and 40) s, respectively. The cultures were radiated by UV and stirred using a bent rod. $5 \mathrm{~mL}$ of culture treated by UV was transferred to a plate for kill rate assaying. The effectiveness of the positive mutation for strain was determined by the acetic acid yield at the same condition.

\section{Spectral determination}

UV-vis spectra were recorded on a Varian CARY 1E UV-vis spectrometer. FTIR spectra were recorded on a Bruker VECTOR22 FTIR spectrometer with a resolution of $1 \mathrm{~cm}^{-1}$ at $298 \mathrm{~K}$ in the range from $4000 \mathrm{~cm}^{-1}$ to $400 \mathrm{~cm}^{-1}$. The spectrometer possesses auto-align energy optimization and a dynamically aligned interferometer, and is fitted with two constringent $\mathrm{BaF}_{2}$ pellets for the measurement of aqueous solution, an OPUS/IR operator, and IR source. A base line correction was made for the spectra that were recorded in air; and then $15 \mathrm{~mL}$ of solution was used in the FTIR spectrometer in every one of the measurements, and the thin layer of samples is less than typically $2 \mu \mathrm{m}$ in thickness. A scanning electron microscope (SEM, Quanta FEG 650, China) with an accelerating voltage of $20 \mathrm{kV}$ and high magnification transmission electron microscope (HR-TEM, JEM-2100, Japan) with an accelerating voltage of $200 \mathrm{kV}$ were employed.

\section{RESULTS AND DISCUSSION}

\section{Identification of the strain}

\section{SEM results}

The characteristics of CSJ-3 strain prepared by negative staining method were observed on a scanning electron microscope (SEM, S3400, Japan). SEM images (Fig. 1) showed that the cells were single or double ellipses (8-11 $\mu \mathrm{m} \times 40-55 \mu \mathrm{m})$.

\section{Description of the strain}

The colonies of strain CSJ-3 on the solid medium were white circles with the diameter of approximately $1 \mathrm{~mm}$.

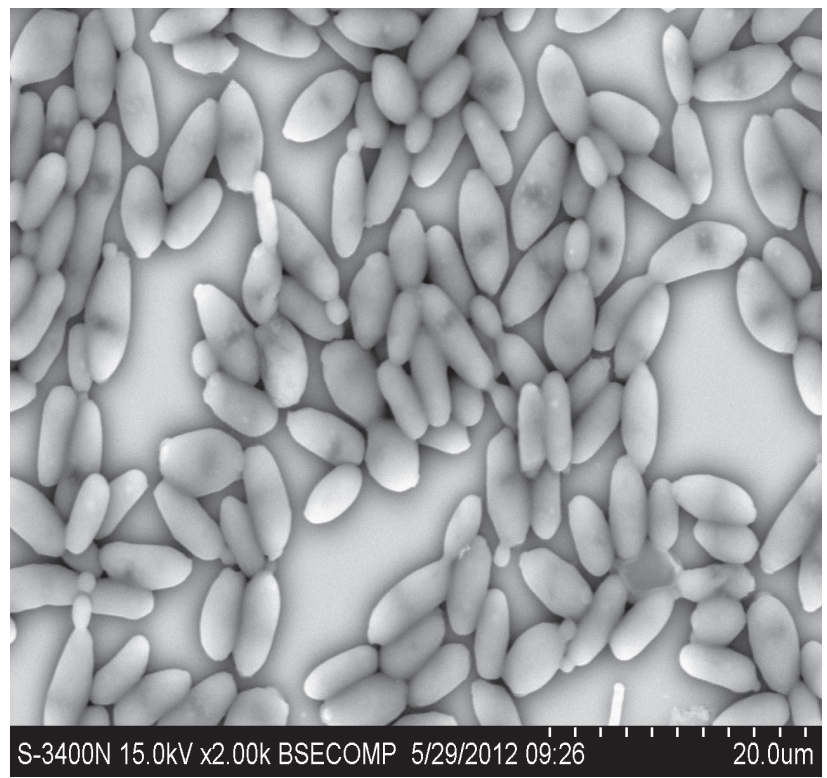

Figure 1. SEM images of CSJ-3 strain

Gram staining test was negative, and a motion experiment showed that the cells could not move in the liquid medium under the microscope. The strain CSJ-3 could utilize glucose, fructose, sucrose, lactose, glycerin, sorbitol, and starch as the carbon source, and could not utilize gluten and citrate as the carbon source. V-P and $\mathrm{H}_{2} \mathrm{~S}$ test results were negative, and contact enzyme and urease test results were positive.

The identification results of 16S rRNA were determined by Shanghai Sangon Biotech. Co., Ltd (China).

The 16S rRNA gene sequence with 1435 bp (GenBank accession number KF861966) was amplified from the genomic DNA of strain CSJ-3 to exhibit $99 \%$ identity with Pseudomonas sp. (Fig. 2). Its physiological and morphological characteristics were also same as Pseudomonas sp. Therefore, CSJ-3 was identified as a new strain of Pseudomonas species.

\section{Optimization of cultivation condition}

\section{Effect of culture temperature on acid production}

At various temperatures, the fermentation conditions were optimized, such as fermentation $\mathrm{pH}=4.5$, ethanol concentration of $7 \%$, glucose as the carbon source, inoculation amount of $32 \mathrm{~mL}$, and rotation speed of 120 $\mathrm{r} / \mathrm{min}$.

Acetic acid could be obtained through CSJ-3 fermentation at the fermentation temperature of 27.0 to $47.0{ }^{\circ} \mathrm{C}$ with an optimal temperature of $37.0^{\circ} \mathrm{C}$ after 30 $\mathrm{h}$ cultivation. The yield of acetic acid revealed an obvious increase with the increase in temperature in the range of 27 to $37^{\circ} \mathrm{C}$ and a decrease at temperatures over $37^{\circ} \mathrm{C}$. The maximum yield of acetic acid was $0.47 \%$ at $37.0{ }^{\circ} \mathrm{C}$ (Fig. 3). 
A G G G G G A G C T A A C C A T G C A A G T C G A G C G G T A G A G A G A A G C T T G C T T C T C T T G A G A G C G C G G A C G G G T G A G T A A T G C C T A $G G$ A A T C T G C C T G G T A $G$ T G G G G A T A A C G T T C G G A A A C G $G$ A C G C T A A T A C C G C A T A C G T C C T A C G G G A G A A A G C A G G G A C C T T C G G G C C T T G C G C T A T C A G A T G A G C C T A G G T C G G A T T A G C T A G T T G G T G A G T A A T G G C T C A C C A A G G C G A C G A T C C G T A A C T G G T C T G A G A G G A T G A T C A G T C A C A C T G G A A C T G A G A C A C G G T C C A G A C T C C T A C G G G A G C A G C A G T G G G G A T A $T$ T G G A C A A T G G G C G A A A G C C T G A T C C A G C C A T G C C G C G T G $T$ G T G A A G A A G G T C T T C G G A T T G T A A A G C A C T T T A A $G$ T $T$ G G G A G G A A G G G T T T A G A T T A A T A C T C T G C A A T T T T G A C G T T A C C G A C A G A A T A A G C A C C G G C T A A C T C T G T G C C A G C A G C C G C G G T A A T A C A G A G G T G C A A G C G T T A A T C G G A A T T A C T G G G C G T A A A G C G C G C G T A G G T G T T T G T T A A G T T G G A $T$ G T G A A A T C C C C G G G C T C A A C C T G G G A A C T G C A T T C A A A A C T G A C T G A C T A G A G T A T G G T A A G G T T G T G G A A T T T C C T G T G T A G C G G T G A A A T G C G T A G A T A T A G G A A G G A A C A C C A G T G G C G A A G G C G A C C A C C T G G A C T A A T A C T G A C A C T G A G G T G C G A A A G C G T G G G A G C A A A C A G G A T T A G A T A C C C T G G T A G T C C A C G C C G T A A A C G A T G T C A A C T A G C C G T T G G A A G C C T T G A G C T T T T A G T G G C G C A G C T A A C G C A T T A A G T T G A C C G C C T G G G G A G T A C G G C C G C A A G G T T A A A A C T C A A A T G A A T T G A C G G G G G C C C G C A C A A G C G G T G G A G C A T G T G G T T T A A T T C G A A G C A A C G C G A A G A A C C T T A C C A G G C C T T G A C A T C C A A T G A A C T T T C T A G A G A T A G A T T G G T G C C T T C G G G A A C A T T G A G A C A G G T G C T G C A T G G C T G T C G C A G C T C G T G T C G T G A G A T G T T T G G G T T A A G T C C C G T A A C G A G C G C A A C C C T T G T C C T T A G T T A C C A G C A C G T A A T G G T G G C A C T C T A A G G A $\mathrm{G}$ A C T G C C G G T G A C A A A C C G G A G G A A G G T G G G A T G A C G T C A A G T C A T C A T G G C C C T T A C G G C C T G G G C T A C A C A C G T G C T A C A A T G G T C G G T A C A G A G G G T T G C C A A G C C G C G A G G T G G A G C T A A T C C C A T A A A A C C G A T C G T A G T C C G G A T C G C A G T C T G C A A C T C G A C T G C G T G A A G T C G G A A T C G C T A G T A A T C G C G A A T C A G A A $T$ G T C G C G G T G A A T A C G T T C C C G G G C C T T G T A C A C A C C G C C C G T C A C A C C A T G G G A G T G G G T T G C A C C A G A A G T A G C T A G T C T A A C C T T C G G G A G G A C G G T A C C A T C G G T G T T T A C G

40

80

120

160

200

240

280

320

360

400

440

480

520

560

$\begin{array}{lll}6 & 0 & 0\end{array}$

640

680

720

760

800

840

880

920

960

$\begin{array}{llll}1 & 0 & 0 & 0\end{array}$

$\begin{array}{llll}1 & 0 & 4 & 0\end{array}$

$\begin{array}{llll}1 & 0 & 8 & 0\end{array}$

$\begin{array}{llll}1 & 1 & 2 & 0\end{array}$

1160

1200

1240

$\begin{array}{llll}12 & 2 & 0\end{array}$

$\begin{array}{llll}13 & 2 & 0\end{array}$

1360

1400

1435

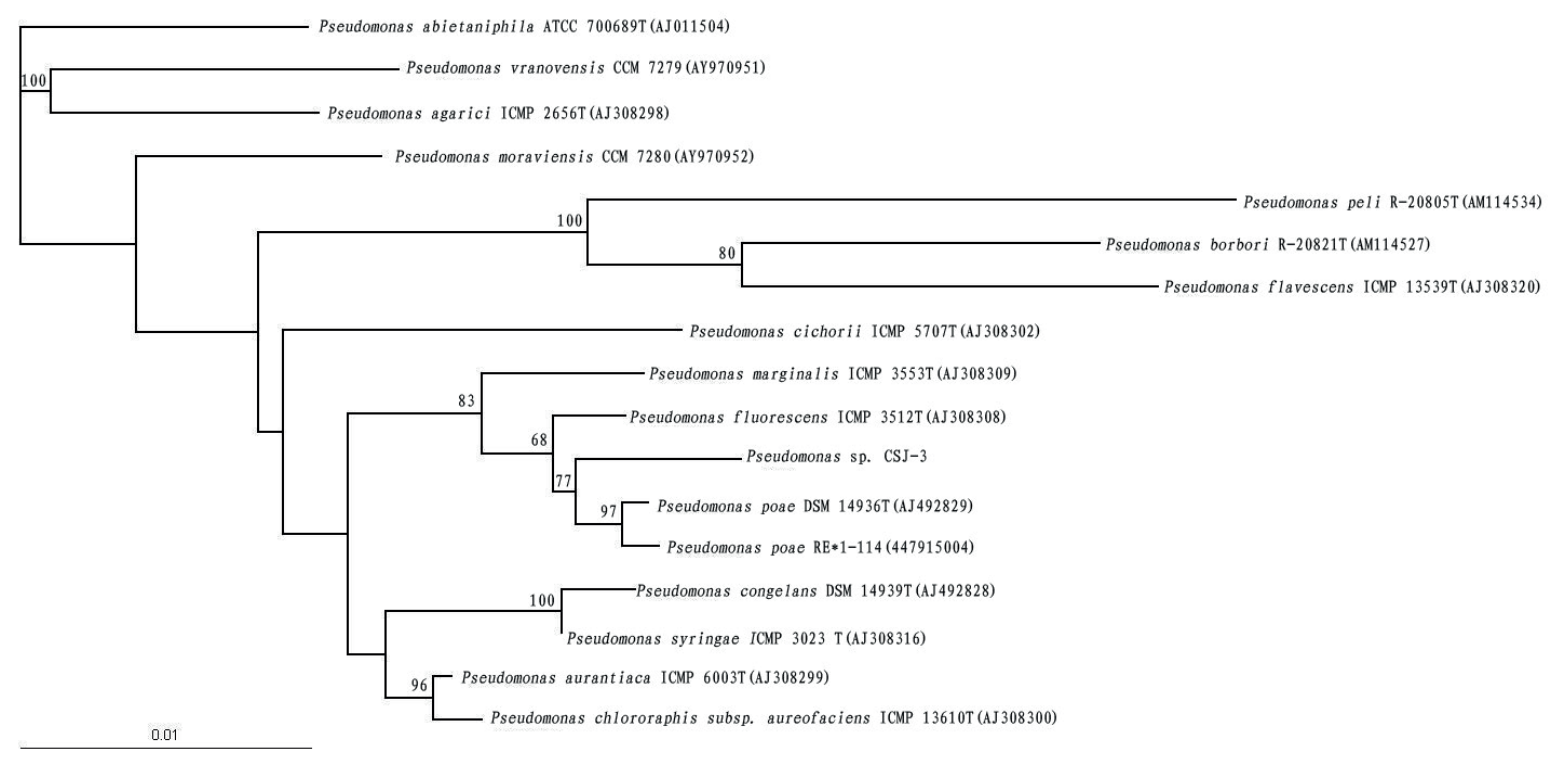

Figure 2. Phylogenetic tree showing the relationship between strain CSJ-3 and related species based on 16S rRNA gene sequences.

The results were attributed to the strain, which plays the commendable role of enzyme at optimum temperature. This phenomenon resulted from the strong enzyme activity at $37.0^{\circ} \mathrm{C}$. At higher temperature, the enzyme in the strain loses its physiological action; at lower temperature, on the contrary, the enzyme in the strain did not have stronger physiological action. 


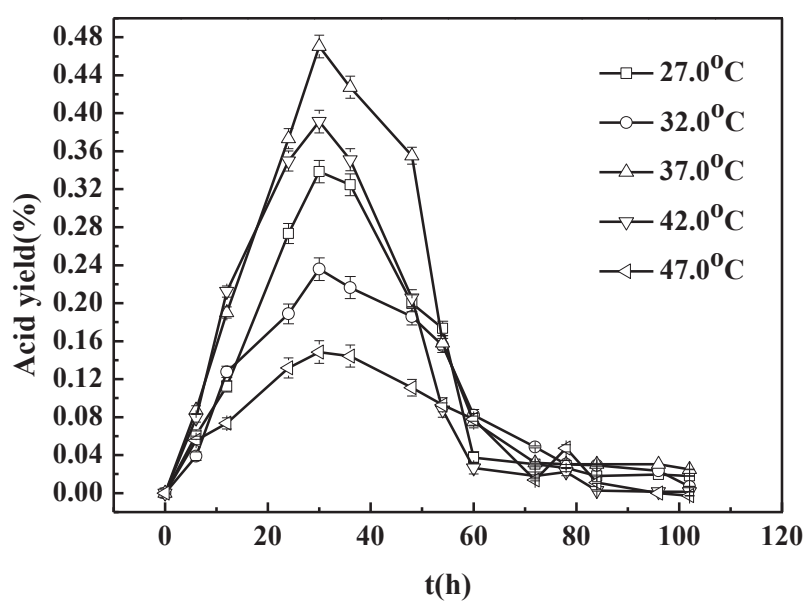

Figure 3. Acetic acid yield of the strain at various temperatures

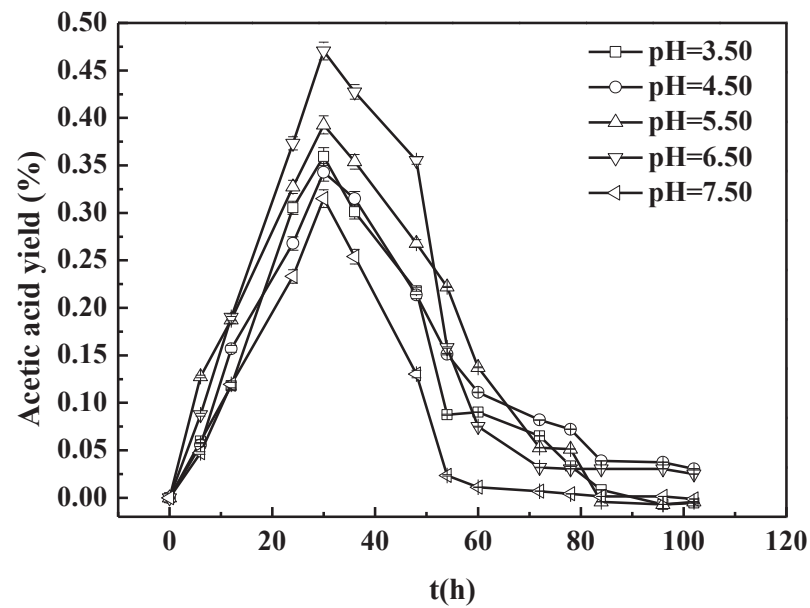

Figure 4. Acetic acid yield of strain at various $\mathrm{pH}$

\section{Effect of initial pH on acid production}

At various initial $\mathrm{pH}$, the fermentation conditions were optimized, such as activated cell solution for $108 \mathrm{~h}$ at 37.0 ${ }^{\circ} \mathrm{C}$, ethanol concentration of $7 \%$, glucose as the carbon source, inoculation amount of $32 \mathrm{~mL}$, and rotation speed of $120 \mathrm{r} / \mathrm{min}$.

When initial $\mathrm{pH}$ was increased from 3.50 to 4.50 , the yield of acetic acid revealed an increase from $0.31 \%$ to $0.47 \%$ after $30 \mathrm{~h}$ cultivation, and then a gradual decrease during the continuous increase in $\mathrm{pH}$ (Fig. 4). These results were consistent with previous reports, in which the yield of acetic acid and cell growth were dependent on initial $\mathrm{pH}$ (Solieri et al., 2008; Trcek et al., 2006).

The results were attributed to the strain that can play the commendable role of enzyme at optimal acetic acidproducing conditions, and can be of benefit for the dissolution of $\mathrm{CO}_{2}$ in culture medium and strain metabolism of the carbon source. At higher $\mathrm{pH}$ condition, the physiological action of acetic acid in cells was affected greatly because the basic complexes entered the cells under the presence of osmotic pressure when the concentration of basic complexes

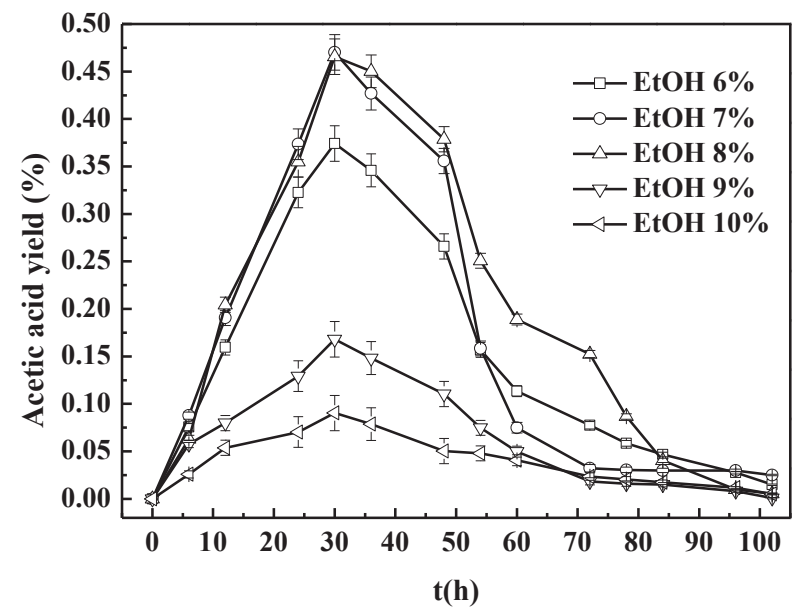

Figure 5. Acetic acid yield of strain in various EtOH concentrations

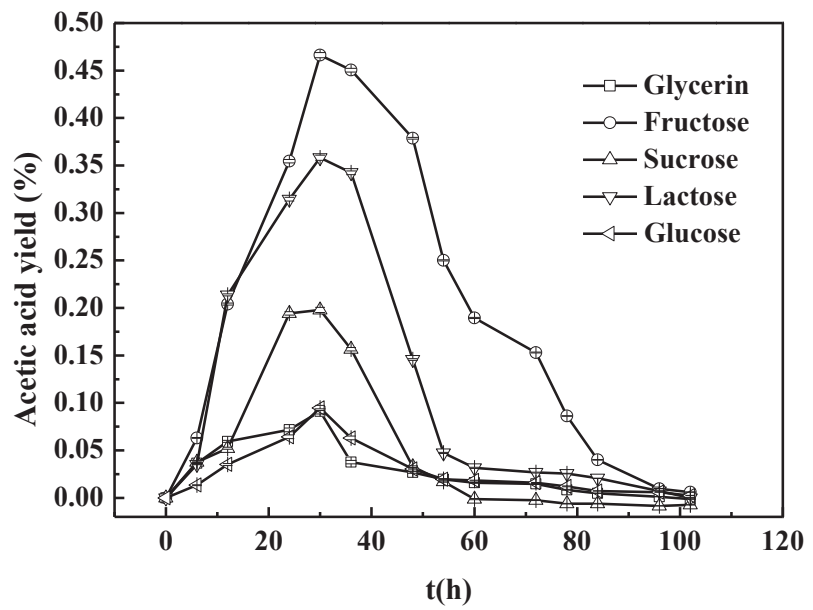

Figure 6. Effect of carbon source on acetic acid production

surrounding cells was higher than in cells. At lower $\mathrm{pH}$, on the contrary, the obviously decreased dissolution capacity of $\mathrm{CO}_{2}$ in the culture medium could affect the growth of the strain. Furthermore, low pH usually results in a lower level of ATP in cells and the inhibition of bacterial growth.

\section{Effect of ethanol concentration on acid production}

At various ethanol concentrations ( $\mathrm{v} / \mathrm{v})$, the fermentation conditions were optimized, such as activated cell solution for $108 \mathrm{~h}$ at $37.0^{\circ} \mathrm{C}$, fermentation $\mathrm{pH}$ of 4.5 , glucose as the carbon source, inoculation amount of $32 \mathrm{~mL}$, and rotation speed of $120 \mathrm{r} / \mathrm{min}$.

The initial ethanol concentrations could affect the production of acetic acid and the growth of CSJ-3 (Fig. 5). The yield of acetic acid revealed an obvious increase with increasing ethanol supplementation (from $6 \%$ to $8 \%$ ) after $30 \mathrm{~h}$, and reached up to a maximum amount of $0.384 \mathrm{~g} / \mathrm{L}$, and then exhibited a gradual decrease.

With various ethanol concentrations, the production of acetic acid showed rapid reduction; for example when the ethanol concentration was $10 \%(\mathrm{v} / \mathrm{v})$ the maximum yield of 
acetic acid was $0.09 \%$. The alcohol oxidase could convert ethanol to acetic acid. At lower ethanol concentration, the oxidase in the strain was plentiful, but the alcohol was lacking. At higher ethanol concentration, the oxidase in the strain could complete its physiological action.

\section{Effect of carbon source on acid production}

At different carbon sources, the fermentation medium was optimized activated cell solution for $108 \mathrm{~h}$ at 37.0 ${ }^{\circ} \mathrm{C}$, fermentation $\mathrm{pH}$ of 4.5 , ethanol concentration of $8 \%$, inoculation amount of $32 \mathrm{~mL}$, and rotation speed of $120 \mathrm{r} /$ min.

The strain can utilize various organic carbon sources, such as glucose, glycerin, fructose, sucrose, and lactose. The strain showed a high yield of acetic acid when glucose was used as the carbon source. When glycerol or lactose was used as the carbon source, the yield of acetic acid was very low, and the maximum yield of acetic acid was $0.095 \%$ and $0.091 \%$, respectively (Fig. 6). Because microorganisms can produce different enzymes, different microorganisms can utilize different carbon sources. This study indicated that the enzyme system of CSJ-3 could better utilize glucose because the utilization rates of other carbon sources were low, especially glycerol and lactose.

\section{Effect of inoculum volume on acid production}

For different bacterial solutions, the fermentation medium was optimized by using activated cell solution for $108 \mathrm{~h}$ at $37.0{ }^{\circ} \mathrm{C}$, fermentation $\mathrm{pH}$ of 4.5 , ethanol concentration of $8 \%$, glucose as the carbon source, and rotation speed of $120 \mathrm{r} / \mathrm{min}$ (Fig. 7).

An inoculum volume in the range of $7-11 \%(\mathrm{v} / \mathrm{v})$ could greatly influence the yield of acetic acid. Fig. 7 showed that the maximum yield of acetic acid was 0.49 $\%$ when $36 \mathrm{~mL}$ of activated bacterial liquid was added to $400 \mathrm{~mL}$ of fermentation medium. The inoculation amount was $9 \%$. Bacteria concentration has an important impact on the growth of strains. In this process, the quantity and compositions of the fermentation medium were unchanged. If the bacterial density was too large, a nutritional deficiency could happen during fermentation process, which could affect the normal growth of bacteria, product synthesis, and too much metabolic waste. Under lower bacterial concentration, on the contrary, the nutritional compounds were rich, so that the production of acetic acid was reduced.

Effect of rotation speeds of the sorting table on acid production

At different rotation speeds, the fermentation conditions were optimized by using activated cell solution for $108 \mathrm{~h}$ at $37.0^{\circ} \mathrm{C}$, fermentation $\mathrm{pH}$ of 4.5 , ethanol concentration of $8 \%$, glucose as the carbon source, and inoculum volume of $36 \mathrm{~mL}$ (Fig. 8).

Fig. 8 showed that the maximum yields of acetic acid were $0.49 \%, 0.50 \%$ and $0.50 \%$, when rotation speeds were $120 \mathrm{r} / \mathrm{min}, 140 \mathrm{r} / \mathrm{min}$ and $160 \mathrm{r} / \mathrm{min}$, respectively. Considering the economic aspects, the rotation speed was optimized as $120 \mathrm{r} / \mathrm{min}$. The rotation speed is a better condition for the dissolution capacity of oxygen and carbon dioxide and the process of mass transfer in culture medium. Higher rotation speed benefits mass transfer of the gases, but not the dissolution of oxygen and carbon dioxide in culture medium. At lower rocking speed, on the contrary, higher dissolution capacity for oxygen and carbon dioxide in culture medium can result in lower dissolution capacity of oxygen and carbon dioxide in culture medium due to a lower mass transfer rate.

\section{Determination of growth curve}

Under the optimal cultivation conditions such as fermentation temperature of $37.0^{\circ} \mathrm{C}$, fermentation $\mathrm{pH}$ of 4.5 , ethanol concentration of $8 \%$, glucose as the carbon source, inoculum volume of $36 \mathrm{~mL}$, and rotation speed of $120 \mathrm{r} / \mathrm{min}$, the strain was cultivated and the growth curve of the strain was plotted, as shown in Fig. 9.

Fig. 9 showed that the lag phase of the strain appeared in the range of 0-6 h, and the adaptation of the strain to the cultivation condition was observed in the lag phase. Exponential phase of the strain was observed within 6-22 $\mathrm{h}$, and the strain could utilize the nutriment in the medium for the growth and rapid proliferation. In the following process, the growth of the strain enters the stationary phase after $22 \mathrm{~h}$.

\section{Mutation breeding}

\section{UV irradiation time}

The inhibitory rate of colonies (UV kill rate) and the positive mutation rate of Pseudomonas CSJ-3 are listed in Table 1.

The longer time could result in the higher killing rate of the strain. The optimal UV radiation time for CSJ-3 was $25 \mathrm{~s}$ and UV killing of CSJ-3 was $83 \%$. Therefore, the mutated cell concentration of $2.8 \times 10 \% \mathrm{~mL}$ was cultured under the conditions as follows: cultivation temperature of $37.0^{\circ} \mathrm{C}$, cultivation $\mathrm{pH}$ of 4.50 , ethanol concentration of 8 $\%$, glucose as carbon source, and rotation speed of $120 \mathrm{r} /$ min. After the cultivation for $30 \mathrm{~h}$, the yields of acetic acid in various mutated strains of CSJ-3 were shown in Fig. 10.

Table 1. UV kill rate of CSJ-3

\begin{tabular}{llllllllll}
\hline Radiation time/s & 0 & 5 & 10 & 15 & 20 & 25 & 30 & 35 & 40 \\
\hline Number of colonies & 305 & 260 & 192 & 125 & 81 & 52 & 33 & 17 & 12 \\
UV kill rate/\% & 0 & 15 & 37 & 59 & 73 & 83 & 89 & 94 & 96 \\
\hline
\end{tabular}




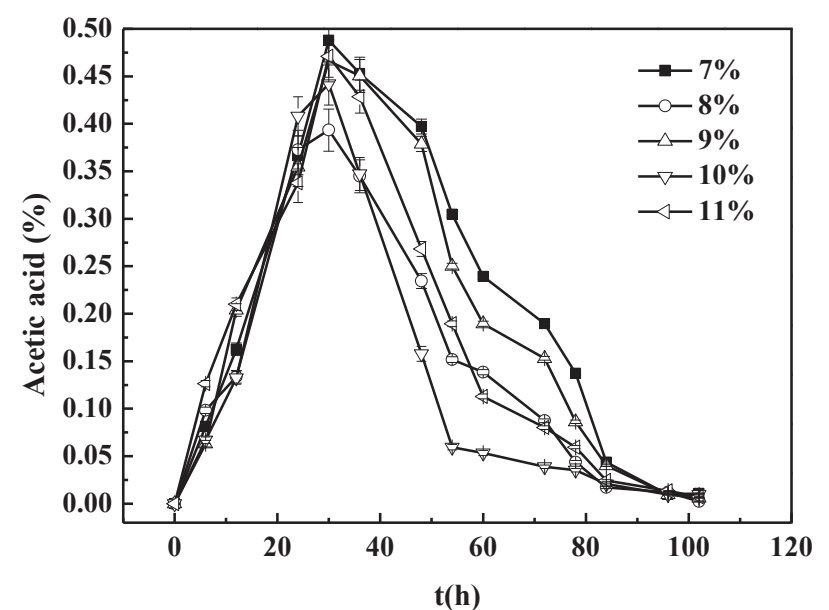

Figure 7. Effect of inoculum volume on acetic acid production

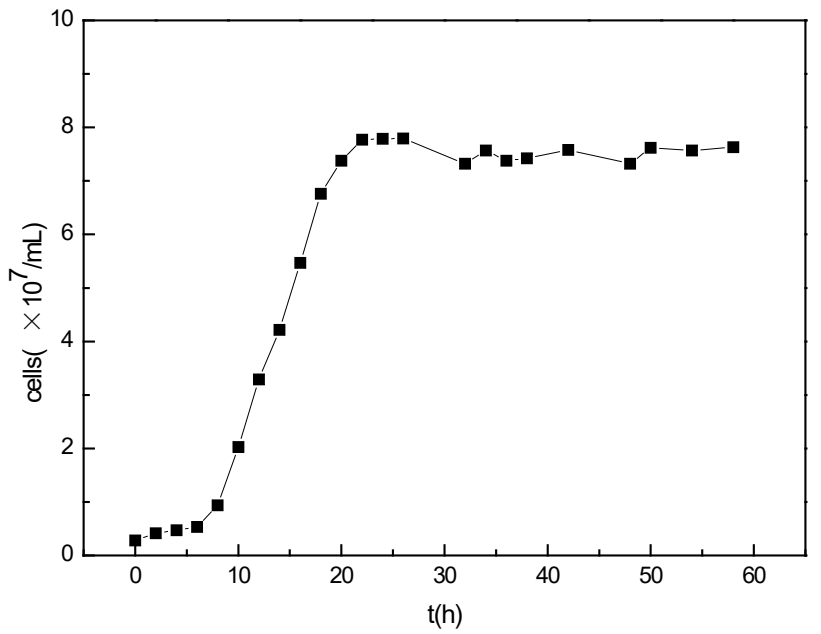

Figure 9. Growth curve of the strain at the optimum condition

\section{Acid producing test}

As shown in Fig. 10, the strains with the serial number of 7,11 , and 15 showed stronger capability to produce acetic acid with $0.51 \%, 0.57 \%$, and $0.58 \%$ when UV radiation duration was $25 \mathrm{~s}$. So the acetic acid producing tests for the serial numbers of 7, 11, 15, and wild strain were explored under the conditions as follows: fermentation temperature of $37.0^{\circ} \mathrm{C}$, fermentation $\mathrm{pH}$ of 4.50 , ethanol concentration of $8 \%$, glucose as carbon source, and rotation speed of 120 $\mathrm{r} / \mathrm{min}$. The acetic acid producing test results were shown in Fig. 11.

In acetic acid producing tests with wild CJS-3 cultivation, Fig. 11 showed that the maximum yield of acetic acid was $0.402 \mathrm{~g} / \mathrm{L}$. But after mutation by UV irradiation, the acetic acid producing activity of CJS-3 (serial number 15) was significantly increased and the maximum yield of acetic acid was $0.59 \%$ with an enhancement of $21 \%$ compared with the yield of acetic acid by CSJ-3.

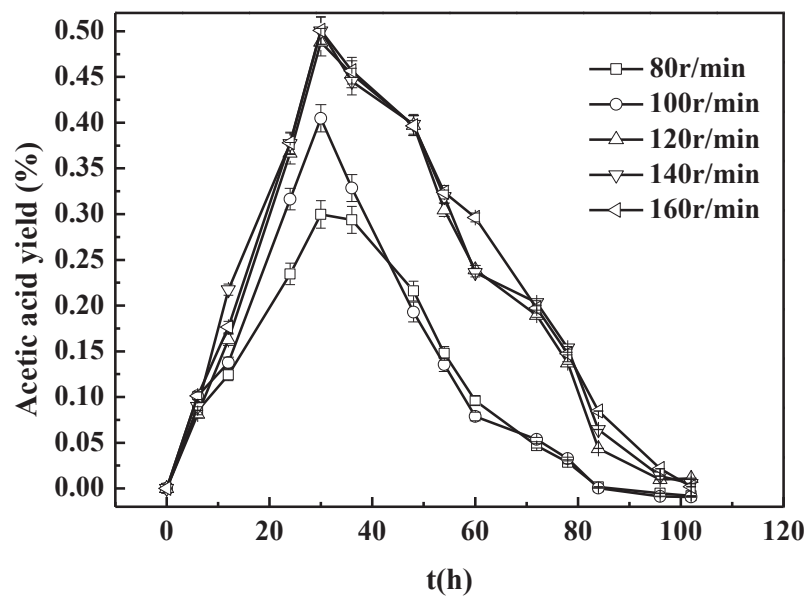

Figure 8. Effect of rotation speeds of the sorting table on acetic acid production

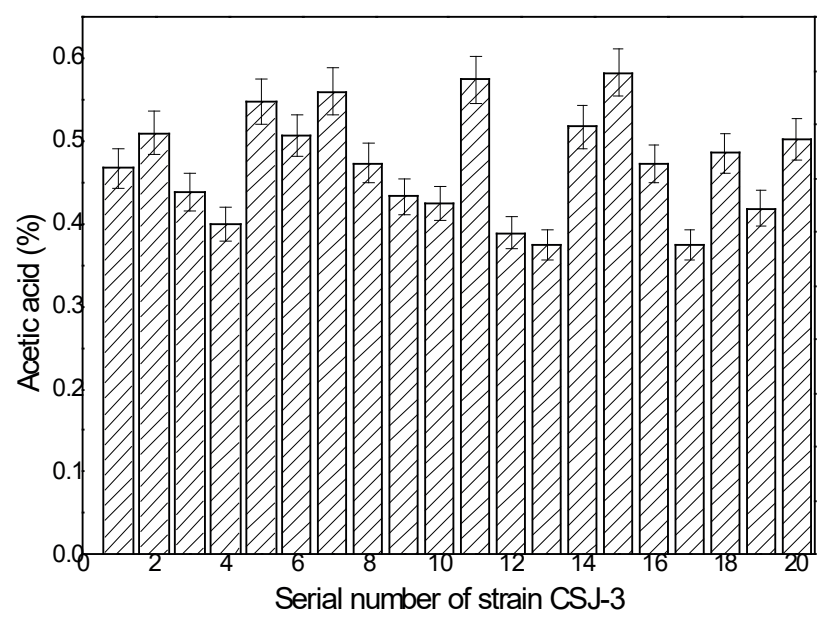

Figure 10. Acetic acid yield of the strains cultured for $30 \mathrm{~h}$ after UV mutagenesis

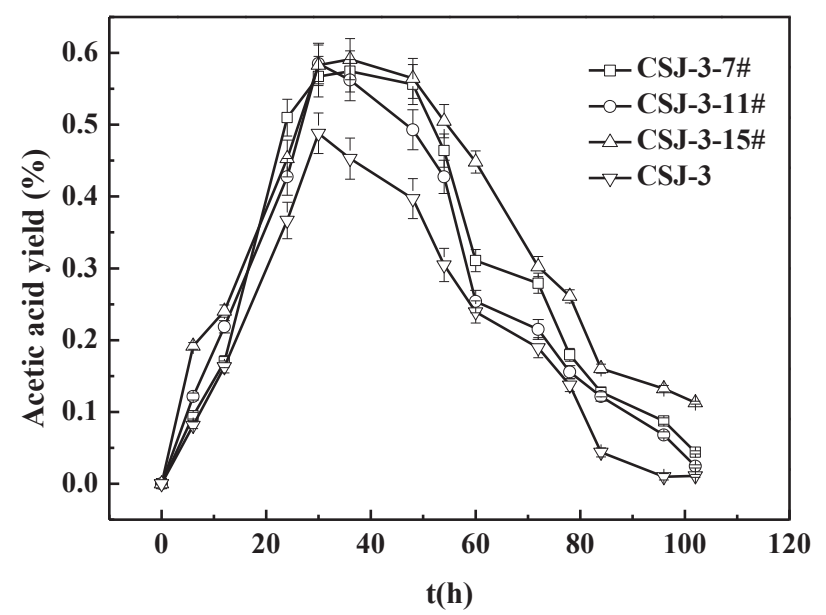

Figure 11. Comparison of acetic acid yield for the mutated strains cultured for three generations and CSJ-3 


\section{Product analysis}

Under the optimum cultivation condition including fermentation temperature of $37.0{ }^{\circ} \mathrm{C}$, fermentation $\mathrm{pH}$ of 4.5 , ethanol concentration of $8 \%$, glucose as carbon source, inoculum volume of $36 \mathrm{~mL}$, and rotation speed of $120 \mathrm{r} / \mathrm{min}$, the strain was cultivated and the fermented solution was confirmed by FTIR and UV spectroscopy.

UV spectra of culture medium, fermented solutions at $0 \mathrm{~h}$ and $30 \mathrm{~h}$, and standard acetic acid were determined as shown in Fig. 12.

Fig. 12 showed the same electronic transition at 190$213 \mathrm{~nm}$. In Fig. 12, the new absorption peak for $30 \mathrm{~h}$ fermented solution was at $260 \mathrm{~nm}$. The absorption band was assigned to the $n \rightarrow \sigma^{*}$ electronic transition of the unshared electronic pair of the oxygen atom in acetic acid

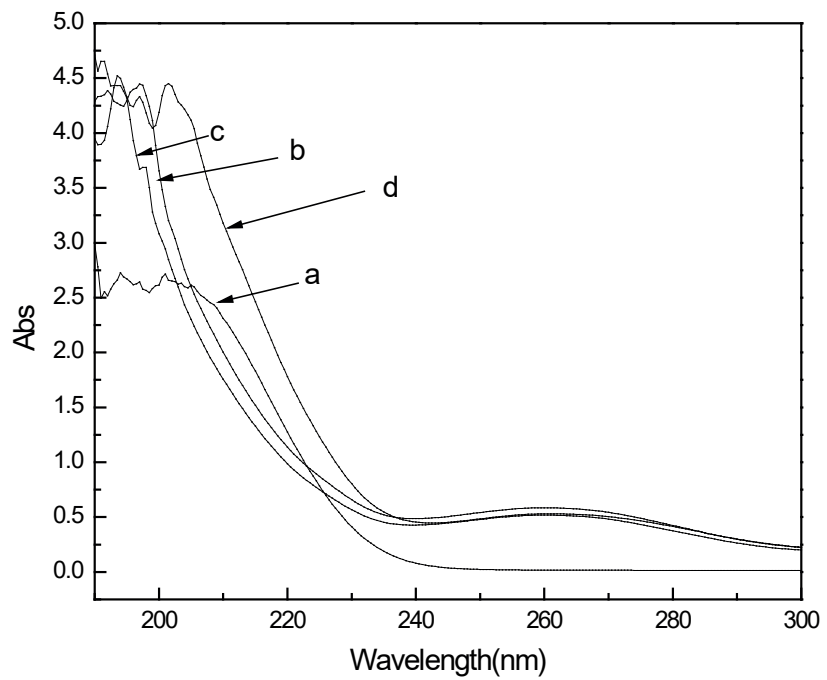

Figure 12. UV spectra of culture medium, fermentative solutions for $0 \mathrm{~h}$ and $30 \mathrm{~h}$, and standard acetic acid. a: pure acetic acid; b: culture medium; c: fermentative solution for $0 \mathrm{~h}$; and $\mathrm{d}$ : fermentative solution for $30 \mathrm{~h}$.

\section{CONCLUSION}

An acid-producing strain was isolated from sludge and identified as Pseudomonas sp. CSJ-3. The optimum cultivation condition was confirmed as follows: fermentation temperature of $37.0^{\circ} \mathrm{C}$, fermentation $\mathrm{pH}$ of 4.5 , ethanol concentration of $8 \%$, glucose amount of $10 \mathrm{~g} / \mathrm{L}$, inoculation amount of $36 \mathrm{~mL}$, and rotation speed of $120 \mathrm{r} / \mathrm{min}$. When ultraviolet irradiation was used for mutation to improve the yield of acetic acid, the yield of acetic acid from the mutated strain reached up to $0.59 \%$, with the enhancement by $21 \%$ compared with the wild strain. because the $n \rightarrow \sigma^{*}$ electronic transition of $\mathrm{H}_{2} \mathrm{O}$ is often observed in the vacuum ultraviolet region, which can be observed in the UV spectrum of acetic acid.

The recorded FTIR spectra of culture medium, fermentative solutions for $0 \mathrm{~h}$ and $30 \mathrm{~h}$, and standard acetic acid were determined as shown in Fig. 13.

In Fig. 13, the new peaks for $30 \mathrm{~h}$ fermented solution were observed at $2610 \mathrm{~cm}^{-1}, 1700 \mathrm{~cm}^{-1}, 1390 \mathrm{~cm}^{-1}$, and $1280 \mathrm{~cm}^{-1}$, which can be observed in the FTIR spectrum of acetic acid $\left(2621 \mathrm{~cm}^{-1}, 1716 \mathrm{~cm}^{-1}, 1398 \mathrm{~cm}^{-1}\right.$, and $\left.1277 \mathrm{~cm}^{-1}\right)$. The peak at $1700 \mathrm{~cm}^{-1}$ can be due to the stretching vibration band of $\mathrm{C}=\mathrm{O}$ (Wu et al., 2013). The peak at $1398 \mathrm{~cm}^{-1}$ can be due to a bending vibrational band of $\mathrm{C}-\mathrm{H}$ (Wu et al., 2013).

Based on UV and FTIR spectral results, the present results show that acetic acid could be detected in $30 \mathrm{~h}$ fermented solution.

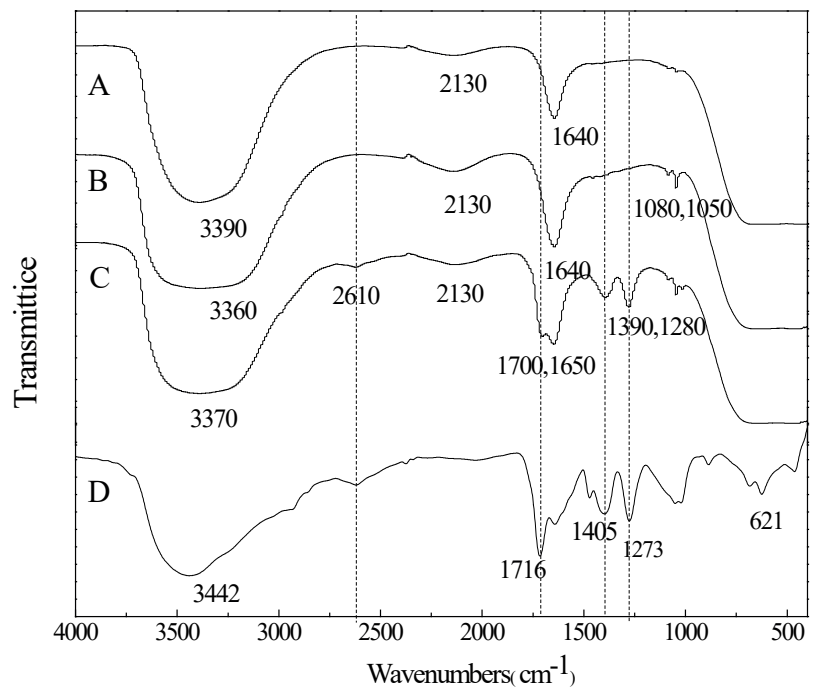

Figure 13. FTIR spectra of culture medium, fermentative solutions for $0 \mathrm{~h}$ and $30 \mathrm{~h}$, and standard acetic acid. A: culture medium; B: fermentative solution for $0 \mathrm{~h}$; $\mathrm{C}$ : fermentative solution for $30 \mathrm{~h}$; and D: pure acetic acid.

\section{ACKNOWLEDGEMENTS}

The Program for Grassland Excellent Talents of Inner Mongolia Autonomous Region, the Program for New Century Excellent Talents in University (NCET-12-1017), Program for Young Talents of Science and Technology in Universities of Inner Mongolia Autonomous Region (NJYT-12-B13), the natural science foundation of Inner Mongolia Autonomous Region (2011BS0601, China), and training plan of academic backbone in youth of Inner Mongolia University of Technology. 


\section{REFERENCES}

Dong, X. Z. Manual of Systemic Determinative Bacteriology (in Chinese). Beijing: Science Press (2001).

Ge, Y., He, J., Zheng, Y., Zhang, L. M., and Zhu, Y. G. Stable isotope probing and its applications in microbial ecology. Acta Ecologica Sinica. 26(5), 1574 -1582 (2006).

Jin, F., Kishita, A., Moriya, T., Enomoto, H., and Sato, N. The production of acetate for use as a roadway deicer by wet oxidation of organic waste, ACS Division of Environmental Chemistry Preprints of Extended Abstracts, Presented at 220th ACS National Meeting, Washington, DC, 40, 73-79 (2000).

Jin, F., Zhou, Z., Kishita, A., Enomoto, H., Kishida, H., and Moriya, T. A new hydrothermal process for producing acetic acid from biomass waste. Chem. Eng. Res. Des. 85, 201-206 (2007).

Kulpa, C. F., Roskey, M., and Mjol, I. Construction of genomic libraries and induction of iron oxidation in Thiobacillus ferrooxidans. Biotechnol. Appl. Biochem. 8, 330-341 (1986).

Lange, M., and Ahring, B. K. A comprehensive study into the molecular methodology and molecular biology of methanogenic Archaea. FEMS Microbiol. Rev. 5(12), 553571 (2001).

McHugh, S., Carton, M., and Mahony, T. Methanogenic population structure in a variety of anaerobic bioreactors. FEMS Microbiol. Lett. 219 (2), 297-304 (2003).

Pratap, C. P., and David, P. C. Stable performance of anaerobic digestion in the Presence of a high concentration of propionie acid. Bioresource Technol., 78, 165-169 (2001).

Schüller, G., Hertel, C., and Hammes, W.P. Gluconacetobacter entanii sp. nov., isolated from submerged high-acid industrial vinegar fermentations. Int. J. Syst. Bacteriol. 50, 2013-2020 (2000).

Sievers, M., Sellmer, S., and Teuber, M. Acetobacter europaeus sp. nov., a main component of industrial vinegar fermenters in central Europe. Syst. Appl. Microbiol. 15, 386-392 (1992).

Sokollek, S.J., Hertel, C., and Hammes, W.P. Description of
Acetobacter oboediens sp. nov. and Acetobacter pomorum sp. nov., two new species isolated from industrial vinegar fermentations. Int. J. Syst. Bacteriol. 48, 935-940 (1998).

Solieri, L., and Giudici, P. Yeasts associated to Traditional Balsamic Vinegar: Ecological and technological features. Int. J. Food Microbiol. 125, 36-45 (2008).

Trcek, J., Ramus, J., and Raspor, P. Phenotypic characterization and RAPDPCR profiling of Acetobacter sp. isolated from spirit vinegar production. Food Technol. Biotechnol. 35, 6367 (1997).

Trcek, J., Raspor, P., and Teuber, M. Molecular identification of Acetobacter isolates from submerged vinegar production, sequence analysis of plasmid pJK2-1 and application in development of a cloning vector. Appl. Microbiol. Biotechnol. 53, 289-295 (2000).

Treck, J., Toyama, H., Czuba, J. Correlation between acetic acid resistance and characteristics of PQQ-dependent $\mathrm{ADH}$ in acetic acid bacteria. Appl. Microbiol. Biotechnol. 70, 366373 (2006).

Treck, J., Mira, N., Jarboe, L. Adaptation and tolerance of bacteria against acetic acid. Appl. Microbiol. Biotechnol. 99, 6215-6229 (2015).

Wang, Z., Yan, M., Chen, X., Li, D., Qin, L., Li, Z., Yao, J., and Liang, X. Mixed culture of Saccharomyces cerevisiae and Acetobacterpasteurianus for acetic acid production. Biochem. Eng. J. 79, 41-45 (2013).

Wu, D., Rasco, B., Vixie, K.R., Ünlü, G., Swanson, B., and Liu, Y. Using Fourier transform infrared (FT-IR) spectroscopy to detect sublethally- or lethally-stressed Listeria innocua treated with acetic acid. LWT-Food Sci. Technol. 54, 456-462 (2013).

Xing, D., Ren, N., Lin, M., and Zhao, L. Ethanoligenens harbinense gen nov., sp. Nov., isolated from molasses wastewater. Int. J. Syst. Evol. Microbiol. 56, 755-760 (2006).

Xu, A. L., Xia, J. L., Zhang, S., Yang, Y., Nie, Z. Y., and Qiu, G. Z. Bioleaching of chalcopyrite by UV-induced mutagenized acidiphilium crytum and acidithiobacillus ferrooxidans. Trans. Nonferrous Met. Soc. China 20, 315-321 (2010) 